e-migrinter

\title{
e-Migrinter
}

$21 \mid 2020$

Famille et migrations

Mobilités, dynamiques frontalières et intégration sous-régionale en zone

CEMAC : le cas des commerçants de Kyé-Ossi

Thèse soutenue le 07 mai 2019 à l'Université de Poitiers

\section{Abêt Mongbet}

\section{(2) OpenEdition}

\section{Journals}

Édition électronique

URL : https://journals.openedition.org/e-migrinter/2548

DOI : $10.4000 /$ e-migrinter.2548

ISSN : 1961-9685

Éditeur

UMR 7301 - Migrinter

Référence électronique

Abêt Mongbet, "Mobilités, dynamiques frontalières et intégration sous-régionale en zone CEMAC : le cas des commerçants de Kyé-Ossi », eMigrinter [En ligne], 21 | 2020, mis en ligne le 29 mars 2021, consulté le 20 mai 2021. URL : http:// journals.openedition.org/e-migrinter/2548; DOI : https://doi.org/10.4000/e-migrinter.2548

Ce document a été généré automatiquement le 20 mai 2021

Tous droits réservés 


\title{
Mobilités, dynamiques frontalières et intégration sous-
}

\author{
régionale en zone CEMAC : le cas des commerçants de \\ Kyé-Ossi
}

Thèse soutenue le 07 mai 2019 à l'Université de Poitiers

\section{Abêt Mongbet}

\section{RÉFÉRENCE}

Mongbet, Abêt (2019) Mobilités, dynamiques frontalières et intégration sous régionale en zone CEMAC : le cas des commerçants de Kyé-ossi, Poitiers, Université de Poitiers, 418 p.

\section{Introduction}

Dans un contexte de faiblesse institutionnelle et de pauvreté, certains États africains peinent à honorer leurs accords, à contrôler toutes leurs populations ou satisfaire leurs attentes. De ce fait, le continent fait face à plusieurs dynamiques tant sur le plan politique que socioéconomique. Cet environnement est, de manière générale, générateur de plusieurs types de mobilités et de migrants dits "aventuriers » (Bredeloup, 2013). Dans ce sillage, certains auteurs qualifient le Cameroun de « country on the move " (Teké et Lengha, $2016: 157)$, un contexte donnant lieu à des nouvelles figures des migrants et à de nouveaux enjeux migratoires (Mimche et Kamdem, 2016). Ce pays est situé en Afrique centrale, une zone qui illustre le paysage d'un continent en mutation à travers ses économies disparates, ses dynamiques frontalières, l'échec de la politique d'intégration sous-régionale de la Communauté économique et monétaire de l'Afrique centrale (CEMAC) (voir la carte $\mathrm{N}^{\circ} 1$ ci-jointe) et notamment le mirage de la libre circulation vieille de cinq décennies (Mongbet, 2018a). Cet échec se matérialise dans le décalage entre les discours politiques et les pratiques de terrain. À la triplefrontière (Cameroun-Gabon-Guinée équatoriale) de Kyé-Ossi, le paradoxe «Plus de 
textes-Moins d'applications » est bien observé. Le blocus frontalier du Gabon et de la Guinée Equatoriale y a produit un système de mobilités et un cadre de dynamiques sociospatiales transfrontalier particulier qui témoigne de la complexité des rapports espace - famille - individus migrants. Ces relations riches de sens ont été travaillées dans le cadre de la recherche doctorale que j'ai menée pendant trois années et demie et soutenue le 07 mai 2019 au laboratoire MIGRINTER de l'Université de Poitiers. L'étude a essayé de comprendre si la continuité territoriale produite par la dynamique frontalière et le système de mobilités en place entrainent l'ouverture des frontières, considérée comme socle de l'intégration sous-régionale, ou au contraire renforcent plutôt leur herméticité. Elle questionne en détail l'évolution de la frontière en rapport avec les échanges d'abord, ensuite le mode de vie des migrants et la formation sociospatiale à Kyé-Ossi, et enfin le champ de mobilités couvert par les commerçants, ainsi que leur effet sur le processus d'intégration sous-régionale. Soutenant l'hypothèse d'ouverture des frontières par le système de mobilités et la continuité territoriale transfrontalière, j'ai réalisé cette étude par une méthodologie intégrant une enquête quantitative auprès de 186 migrants, une enquête qualitative à travers des entretiens dont 31 auprès des personnels des Institutions des États du Cameroun, du Gabon et de la Guinée équatoriale et des autorités de Kyé-Ossi, 116 en contexte d'immersion auprès des migrants, et 6 récits biographiques. À ces sources primaires ont été adjointes les images satellitaires pour mieux apprécier l'évolution sociospatiale à une échelle globale des trois frontières.

\section{Carte $\mathrm{n}^{\circ} 1$ : Localisation de Kyé-Ossi}

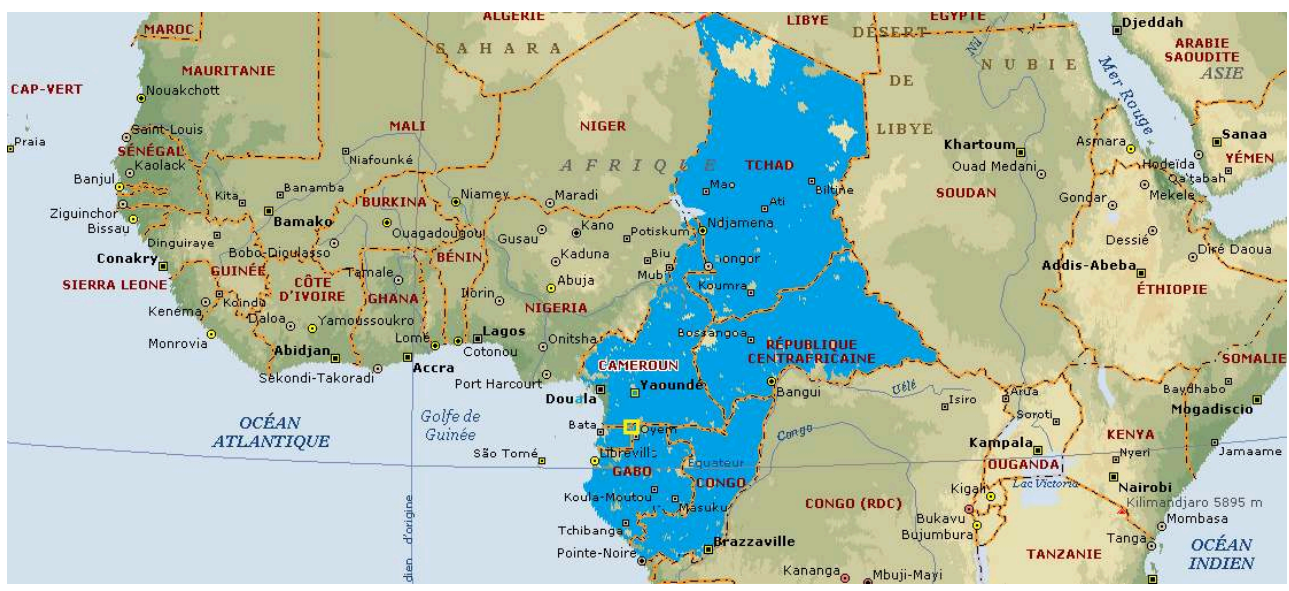




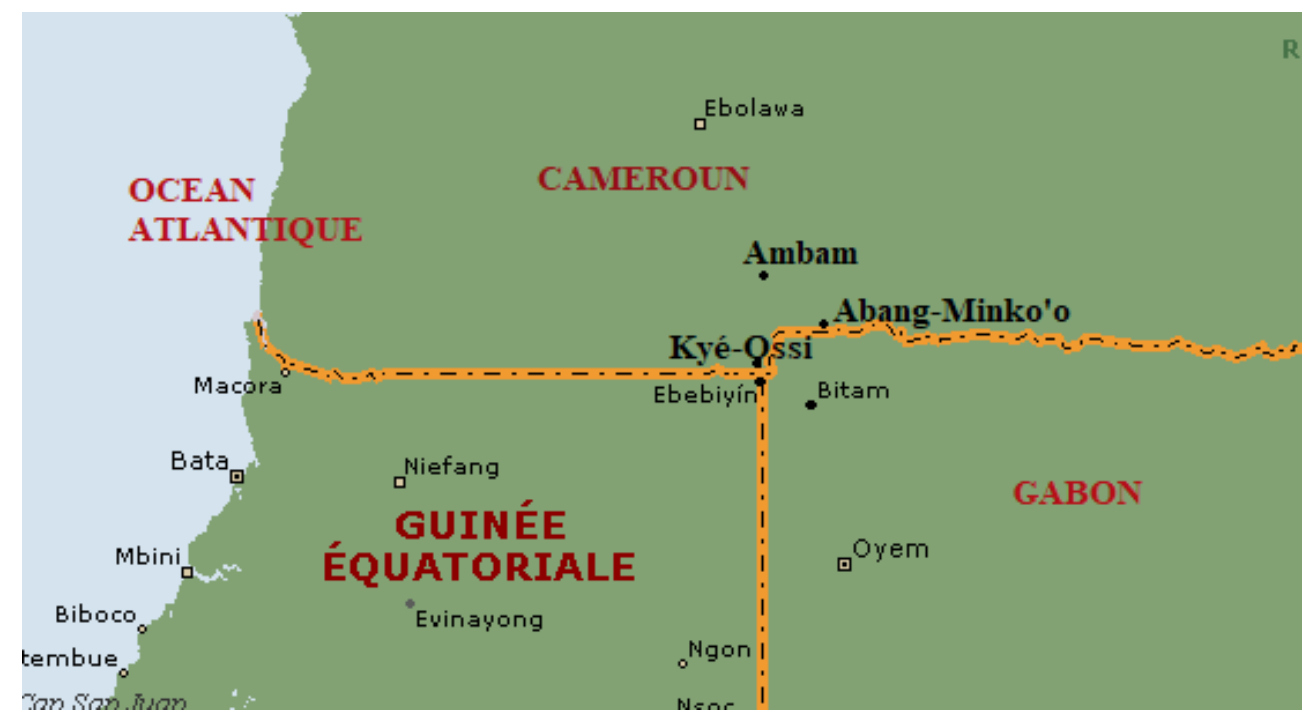

En bleu, les pays de la CEMAC. Le petit cadre jaune entoure la frontière commune entre les trois pays (triple frontière) Cameroun, Gabon, Guinée équatoriale. Le zoom montre la position de Kyé-Ossi à la triple frontière et d'autres villes frontalières. La frontière internationale est en orange.

Source : Réalisation de l'auteur à partir de Encarta.

\section{Le peuplement migratoire de la triple-frontière : des individus aux familles}

L'acte $\mathrm{N}^{\circ}$ 1/72-UDEAC-70-A du 22 décembre 1972 relatif à la convention commune sur la libre circulation des personnes et le droit d'établissement dans l'Union douanière et économique de l'Afrique centrale (UDEAC) constitue la première référence du processus de libre circulation en zone CEMAC. La dernière pouvant être citée est la circulaire $\mathrm{N}^{\circ} 0001 \mathrm{MISL} / \mathrm{MDN}$ du Gabon relative à la libre circulation, signée le 19 octobre 2017 par le Ministre gabonais de l'Intérieur et de la Sécurité, Chargé de la Décentralisation et du Développement Local (MISDL) et le Ministre de la Défense Nationale (MDN). Cette circulaire précise :

«Tout ressortissant des 5 autres pays de la CEMAC détenteur d'un passeport d'une validité d'au moins trois mois, n'est plus soumis à l'obligation du visa d'entrée au Gabon pour tout séjour ne dépassant pas trois mois (90 jours)».

Entre ces deux dates, les accords, les traités et les directives pour la libre circulation des personnes et des biens sont nombreux entre les six pays de la CEMAC (Cameroun, Congo, Gabon, Guinée équatoriale, RCA, Tchad). Cependant, le Gabon et la Guinée équatoriale, respectivement quarante-huitième et cinquante-quatrième pays africains en termes d'ouverture aux immigrants africains, sont les plus mauvais candidats dans ce processus de libre circulation. L'imposition du visa aux ressortissants de la sousrégion pour toute entrée sur leurs territoires justifie leur blocus frontalier à Kyé-Ossi. Cette hostilité à l'entrée du territoire, qui est contradictoire aux textes de la CEMAC, fait de cette triple-frontière une barrière aux mobilités internationales des Camerounais et à l'intégration sous-régionale.

4 Face à leur situation socioéconomique insatisfaisante, les Camerounais ont eu comme alternative, entre autres, la migration afin de trouver un avenir meilleur. Se faisant, Kyé-Ossi s'est affirmé progressivement comme une destination particulière. Dans cette partie du pays et ses environs, les migrations de la deuxième moitié du 20e siècle ont 
succédé à celles de la fixation des ethnies dites autochtones de nos jours au Cameroun. D'abord un lieu de migration par réseau communautaire et difficile d'accès, cette partie du territoire devient fonctionnelle à partir de 2003 grâce à l'aménagement du territoire par l'État (Carte $\mathrm{n}^{\circ} 2$ ci-dessous). À la faveur du développement des voies de communication dont le bitumage de la route et la construction du pont sur le fleuve Ntèm qui l'isolait de l'Hinterland, cette frontière va connaitre un accès facile qui déjoue la discrimination xénophobe des autochtones Ntumu. Dès lors s'offre l'opportunité aux free migrants plus nombreux de suivre les primo migrants déterminés. Les premiers désignent les migrants qui arrivent à Kyé-Ossi après l'aménagement des voies de communication facilitant l'accès, contrairement aux seconds qui ont atteint la frontière dans des conditions difficiles. Ces derniers sont aussi suivis par les membres de leurs familles pour peupler la frontière. Ainsi, 85 \% des migrants arrivent à la frontière après 2003.

Graphique $\mathrm{n}^{\circ} 1$ : Évolution de la densité de Population

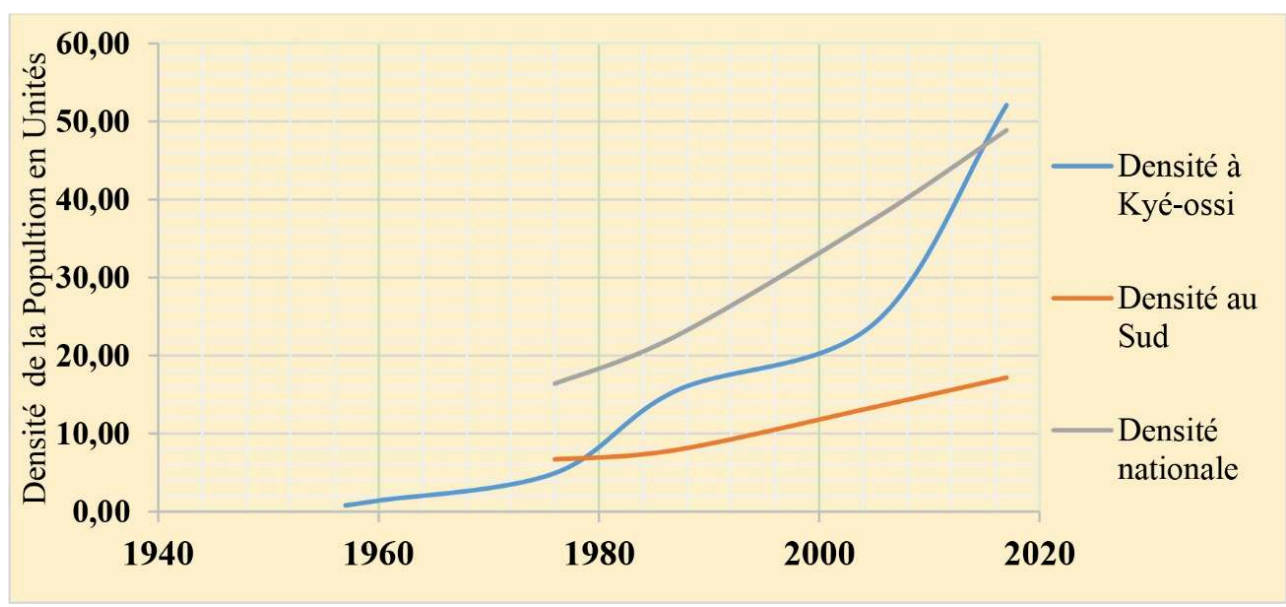

Source : Mongbet, 2019 :p. 197 (Sud : région du Cameroun abritant Kyé-Ossi)

C'est cette poussée démographique qui rend les migrants majoritaires par rapport aux autochtones. 
Carte $n^{\circ} 2$ : Étatisation du territoire vers la frontière (Kyé-Ossi) Gabon-Guinée équatoriale

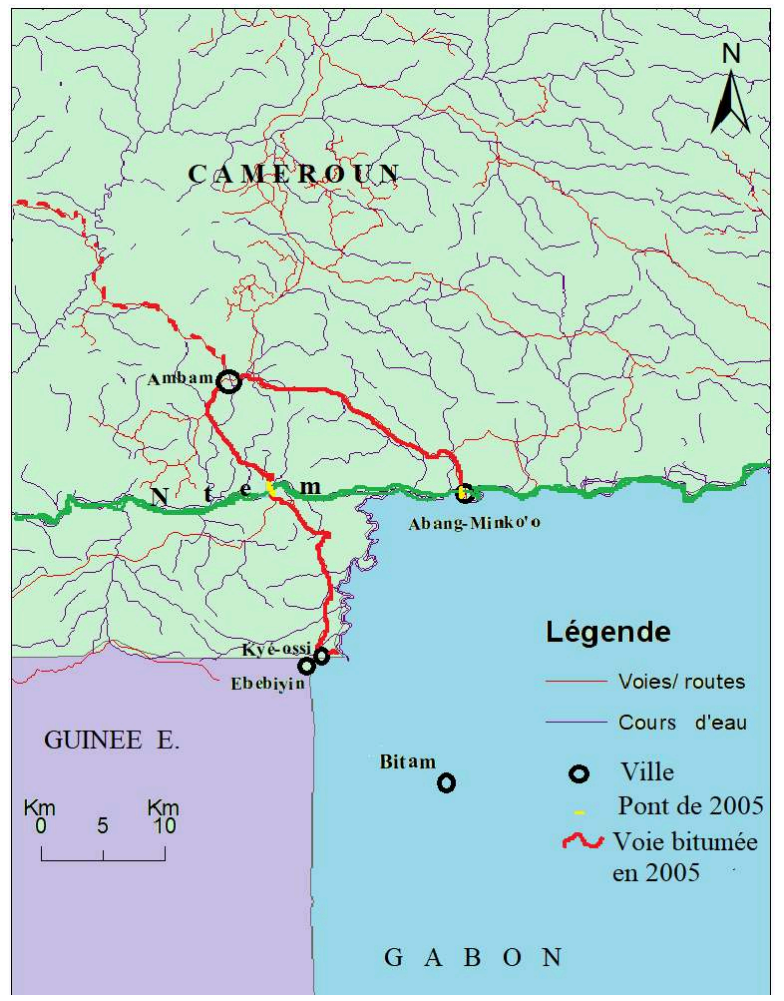

Le fleuve Ntem en vert a été traversé par deux ponts qui accompagnaient le bitumage de la route. Source : Mongbet, 2019 : p. 193.

6 L'évolution de la densité humaine à Kyé-Ossi (Graphique $\mathrm{n}^{\circ} 1$ ci-dessus) montre deux sursauts dus au peuplement migratoire qui l'affecte dans deux périodes : 1976-1987 et 2003-2017. L'enquête révèle que 38,48 \% des femmes y sont arrivées grâce au mariage et $93 \%$ sont accueillies par leurs maris qui les avaient devancées. Le deuxième sursaut de la courbe s'explique non seulement par les arrivées massives, mais les regroupements familiaux internes et les naissances issues des migrants. En moyenne, la taille moyenne de ménage chez les migrants est passée d'environ deux personnes $(2,44)$ à cinq $(4,9)$ entre 2008 et 2017. Ce doublement provient des accouchements et du regroupement familial qui valent respectivement $66 \%$ et $15 \%$ des raisons d'augmentation du nombre de personnes dans les ménages. Les données décrivent donc une forme de transition dans le peuplement migratoire de Kyé-Ossi: de l'individu à la famille. Dans ces mouvements humains heurtés à la traversée de la frontière, la majorité des migrants viennent de l'ouest du Cameroun, notamment du groupe ethnique Bamoun à dominance musulmane. Leurs installations et insertions économiques sont plus l'œuvre des efforts individuels et d'aide familiale selon mes données. Vu leur prédominance dans les activités économiques et les réalisations immobilières, le mode de vie que ces migrants en général y développent reflète véritablement leur domination numérique au sein de la population de Kyé-Ossi, faisant subir aux natifs une marginalisation sociospatiale. 


\section{Kyé-Ossi dans la dynamique sociospatiale}

7 La densité de la population à Kyé-Ossi a stimulé progressivement celle des activités économiques. Avant l'arrivée des migrants, le commerce était réduit aux produits de la nature forestière de l'environnement immédiat. Il s'est intensifié progressivement en se diversifiant pour acquérir finalement une dimension sous-régionale (Graphique $\mathrm{n}^{\circ} 2$ ). Cette dynamique commerciale a évolué conjointement avec l'acquisition foncière, les réalisations immobilières individuelles et communautaires telles que les logements, les boutiques, les foyers culturels, les mosquées et les églises. De telles structures participent à la construction d'une nouvelle identité sociospatiale (Mongbet, 2018b). Simultanément, l'État accentue la construction de l'espace à travers certaines infrastructures de base telles que les écoles, l'hôpital, les sièges des services administratifs, des forces de contrôle et de sécurité. Toutefois, les structures de santé et les établissements scolaires privés bâtis par les migrants y sont bien plus nombreux que ceux de l'État. Ils répondent aux besoins de la descendance des migrants en pleine croissance : jeunes et enfants devant se soigner et aller à l'école. L'agrandissement de famille s'avère ainsi l'un des moteurs principaux de la transformation sociospatiale observée à Kyé-Ossi.

Graphique $n^{\circ} 2$ : Évolution des exportations des produits vivriers à Kyé-Ossi

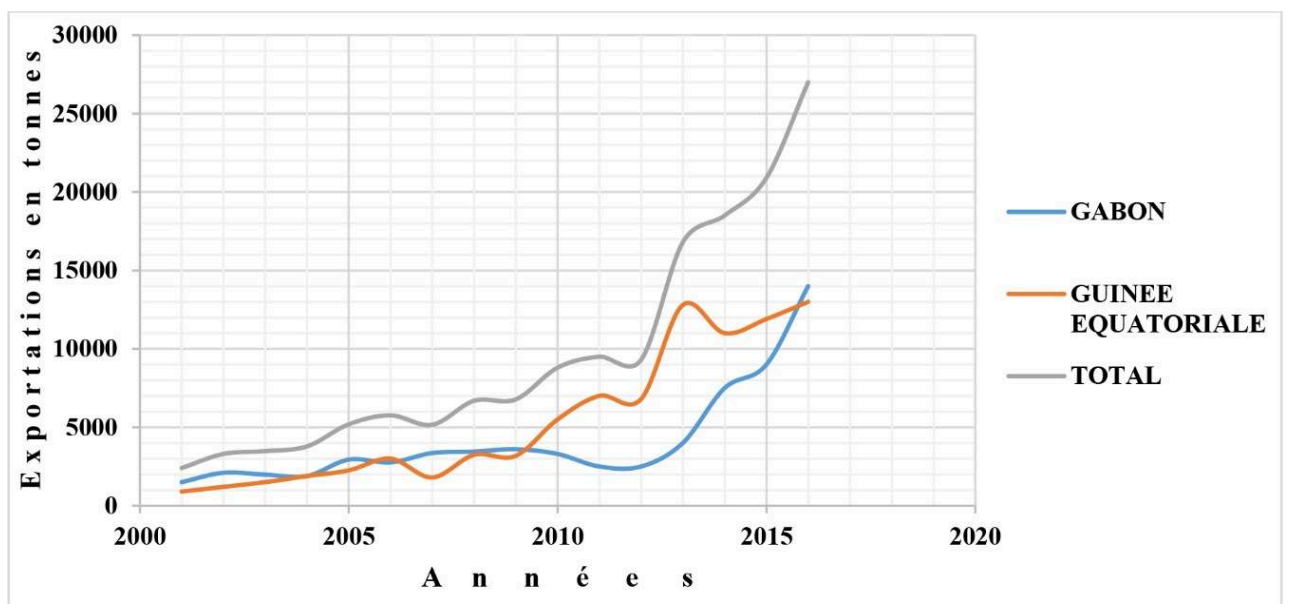

Source : Mongbet, 2019 : p. 207.

$8 \mathrm{Au}$ fur et à mesure que les migrants internes transformaient Kyé-Ossi, les villes frontalières d'Ebebiyin et de Bitam évoluaient en matière de constructions et d'activités humaines. Cette évolution impulsée par les migrants et leurs activités commerciales a engendré une sorte de continuité territoriale transfrontalière, laquelle est attisée par les réseaux de mobilités internationales. 
Carte $n^{\circ} 3$ : Champs de mobilités des migrants commerçants par rapport à la frontière

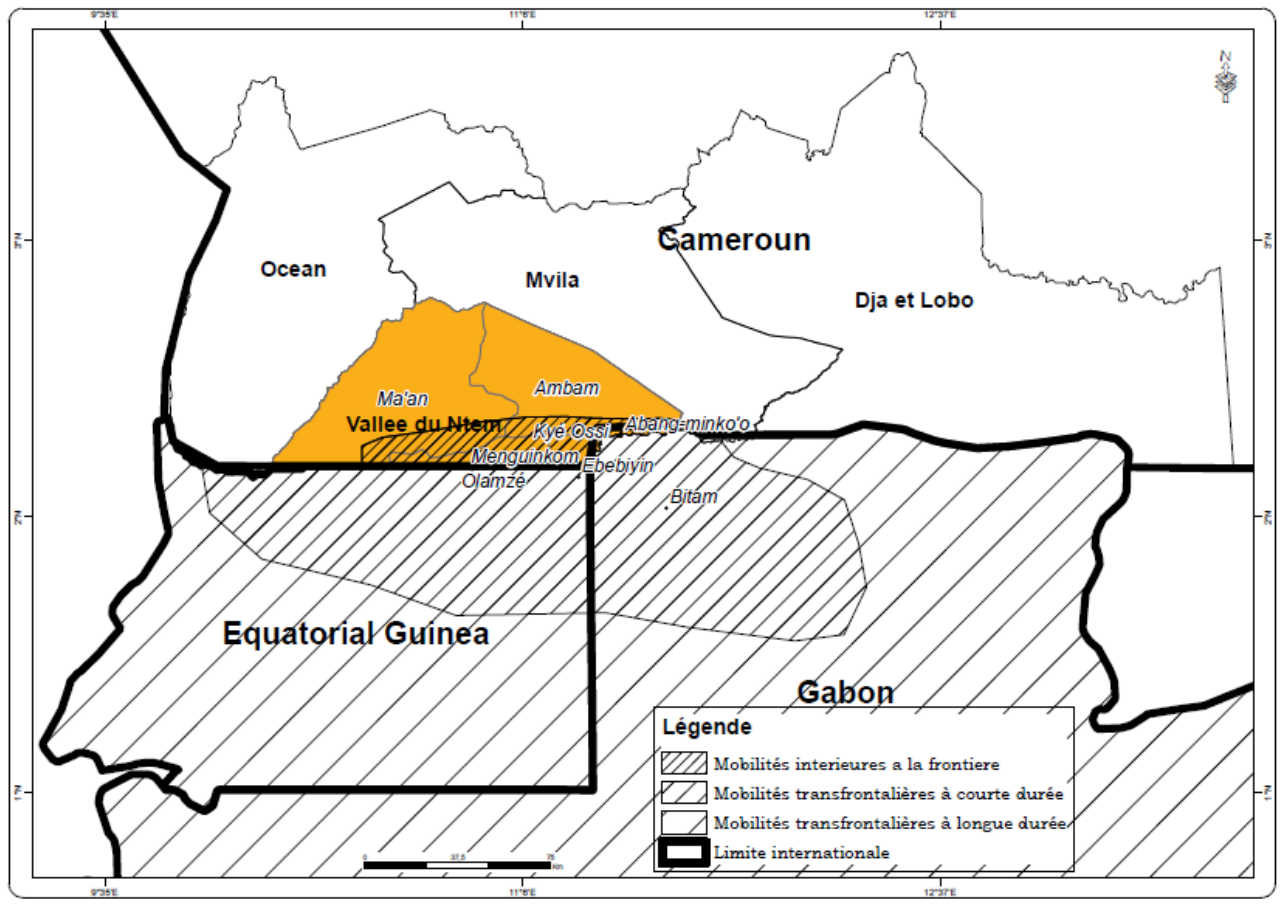

Source : Mongbet, 2019 : p. 315.

\section{Réseaux, mobilités internationales et citoyenneté sous-régionale}

Les flux de mobilités observés sont façonnés par les multiples liens de solidarité entre les migrants et les populations qui intègrent leurs champs de mobilités. Ils s'expriment de diverses manières et sur plusieurs dimensions. Cette typologie permet de

«mettre en évidence des dynamiques de transformation de la fonction sociale des espaces dans le temps » (Morange et Schmoll, $2016:$ p. 161).

Dans le triangle frontalier Kyé-Ossi-Ebebiyin-Bitam qui interfère avec la frontière commune des trois pays, les familles biologiques se sont installées différemment dans une logique de conquête spatiale. Leur schéma de circulation s'assimile à celui des autochtones Ntumu dont la communauté transfrontalière dilue le caractère étatique de la frontière en tant que limite de souveraineté. À cet effet, la répartition des familles autour de la frontière met en lumière trois cas. Le premier concerne les familles migrantes bloquées entièrement par la frontière et divisées par la migration interne jusqu'à Kyé-Ossi. Le deuxième porte sur les familles divisées par la frontière et contenant plusieurs ménages. Ce type est surtout observé entre Bitam et Kyé-Ossi tandis que du côté d'Ebebiyin, on assiste plutôt à des groupes d'amis séparés par la frontière. Dans cette catégorie, la frontière guinéenne joue un rôle de filtre en déqualifiant les femmes, car les témoignages de terrain tendent à monter qu'elles évitent de traverser la frontière, conscientes des peines et des traitements malveillants réservés aux étrangers. Ainsi, le mari est en mobilité internationale jusqu'à Bata en territoire guinéenne tandis que la femme garde les enfants à la frontière à Kyé-Ossi. La troisième figure est celle des familles spatialement réunies, constituées en réalité des 
ménages communs, soit à Kyé-Ossi, soit à Bata. Entre ces différents schémas familiaux émerge le capital spatial qui facilite les mobilités commerciales et anime les réseaux d'immigration des camerounais de Kyé-Ossi. L'installation multi-située des membres de familles contribue alors à dompter l'espace et la frontière, à dynamiser les réseaux où les primo-migrants par leur longue expérience spatiale et leur intégration linguistique, s'arrogent une identité territoriale et relationnelle entre les trois pays. En conséquence, les médiateurs frontaliers comportent autant ces acteurs informels que ceux formels reconnus officiellement par les États voisins. Leur hiérarchisation au-delà de la frontière et les traitements subis par les camerounais en territoire étranger questionnent la citoyenneté sous-régionale en zone CEMAC.

11 Pour tout passage aux frontières du Gabon et de la Guinée, le mirage de libre circulation persiste avec une sorte de procès d'intention d'immigrer fait contre les camerounais. En plus, les actes subis par ces camerounais traduisent une intégration différenciée et des schémas d'accueil différents entre les deux pays voisins du Sud. Mener une vie politique en toute quiétude, pratiquer le commerce et l'espace sans obstacle xénophobe, réaliser et se mouvoir sans être interpelé du fait d'être camerounais, tels sont les éléments d'expression d'une vie citoyenne. Seulement, les populations de la ville frontalière de Bitam s'avèrent plus pratiques et plus accueillantes que celles d'Ebebiyin. La frontière linguistique renforce chez les guinéens la frontière sociale, réduisant par conséquent les marges de liberté et de réalisations chez les immigrants camerounais sur le territoire gabonais. Ces nuances traduisent une citoyenneté sous régionale différenciée entre les pays de la CEMAC signataires des mêmes traités de libre circulation. Face à la sécurisation de l'immigration qui ressort de l'analyse, le mirage d'ouverture frontalière s'alimente de la multiplicité d'acteurs formels et de l'ajustement des stratégies des migrants. Dans ce contexte, le schéma de Ghost town plane sur la dynamique sociospatiale à Kyé-Ossi.

\section{Conclusion}

12 La frontière commune Cameroun-Gabon-Guinée équatoriale est un espace en mutation caractérisé par un peuplement migratoire qui a progressé des individus aux familles. Son évolution s'accompagne des mobilités transfrontalières que ces familles situées de part et d'autre développent. La ville frontalière de Kyé-Ossi au Cameroun est un point satellite de ces mobilités au regard de son impulsion en tant que lieu de première installation, de passage, ou de construction de réseaux par les mouvements transfrontaliers des camerounais migrants. L'hypothèse d'ouverture des frontières à travers l'absence des renvois des camerounais sans visa s'est infirmée au fil de l'analyse. Elle révèle une pluralité d'acteurs médiateurs frontaliers, une position multisituée des familles pour contourner la barrière frontalière et un traitement différencié des migrants qui déjoue l'idée de libre circulation tant prônée dans les discours et traités étatiques. Entre manque de pragmatisme des autorités et stratagèmes des migrants, la réflexion au gré du temps s'enrichit des faits politiques, économiques, sociaux, culturels tant nationaux que sous-régionaux. 


\section{BIBLIOGRAPHIE}

Toutes les références sont citées dans la thèse et n'ont plus fait l'objet d'une nouvelle exploitation.

Bredeloup, Sylvie (2013) Les temps du transit dans la migration africaine, Le journal des africanistes, vol. 83, $\mathrm{n}^{\circ} 2$, pp. 58-90.

Mimche, Honoré ; Kamdem, Pierre (dir) (2016) Emigration internationale au Cameroun; des nouveaux enjeux aux nouvelles figures, Paris, L'Harmattan, $484 \mathrm{p}$.

Mongbet, Abêt (2019) Mobilités, dynamiques frontalières et intégration sous régionale en zone CEMAC : le cas des commerçants de Kyé-ossi, Poitiers, Université de Poitiers, 418 p.

Th. Doct. : Géographie humaine : Université de Poitiers : 2019.

Mongbet, Abêt (2018a) Intégration sous régionale et libre circulation en zone CEMAC. Des déclarations médiatiques aux pratiques frontalières : quel gap ?, Quatrième édition du Colloque International d'Agadir « Migrations, mobilités, frontières : des représentations aux traitements médiatiques ", Agadir- Maroc du 25 au 27 avril 2018.

Mongbet, Abêt (2018b) Le Cameroun entre intégration nationale et autochtonie : exemple du patrimoine territorial entre les Ntumu et les migrants à Kyé-Ossi, communication aux 4èmes journées scientifiques de la FALSH «Patrimoine(s), Mémoire(s) et Identité(s) en Afrique », 6-8 juin 2018 à l'université de Maroua.

Morange, Marianne ; Schmoll, Camille (2016) Les outils qualitatifs en géographie : méthodes et applications, Paris, Armand Colin, $224 \mathrm{p}$.

Teke Takwa, Johnson ; Lengha, Tohnain Norbert (2016) A country on the move : new figures of emigrants in Cameroon, In Mimche,H. ; Kamdem, P. (dir) Émigration internationale au Cameroun ; des nouveaux enjeux aux nouvelles figures, Paris, L'Harmattan, pp. 157-178.

\section{RÉSUMÉS}

À cause du blocus frontalier imposé par le Gabon et la Guinée équatoriale au niveau de la frontière commune avec le Cameroun, les migrants venant de ce dernier s'accumulent à la frontière du côté camerounais à Kyé-Ossi. Cette accumulation engendre une sédentarisation des migrants, leur installation transitaire ou permanente, faisant de la localité une zone de diverses mobilités. Ce faisant, leur présence s'illustre par des mutations socio-spatiales qui transforment cette zone transfrontalière en territoire continu, processus accompagné par des actions étatiques. L'ensemble produit et anime grâce aux familles multisituées et leurs réseaux plusieurs formes de mobilités chez les commerçants qui pratiquent l'espace de manière différenciée. L'étude vise à comprendre si la continuité territoriale que procure cette dynamique transfrontalière assouplira les contrôles aux frontières ou au contraire renforcera leur herméticité.

\section{INDEX}

Mots-clés : libre circulation, frontière, intégration sociale, étude régionale Index géographique : Cameron, Guinée équatoriale 


\section{AUTEURS}

\section{ABÊT MONGBET}

Démographe, cadre au Bureau Central des Recensements et des Études de Population (BUCREP),

Cameroun

abetmongbet@yahoo.fr 